\title{
Centering theory and the Italian pronominal system
}

\author{
Barbara Di Eugenio* \\ Department of Computer and Information Science \\ University of Pennsylvania \\ Philadelphia, PA \\ dieugeni@linc.cis.upenn.edu
}

\begin{abstract}
In this paper, I give an account, in terms of centering theory [GJW86], of some phenomena of pronominalization in Italian, in particular the use of the null or the overt pronoun in subject position. After a general introduction to the Italian pronominal system, I will review centering, and then show how the original rules given in [GJW86] have to be extended or modified. Finally, I will show that centering does not account for two phenomena: first, the functional role of an utterance may override the predictions of centering; second, a null subject can be used to refer to a whole discourse segment. This latter phenomenon should ideally be explained in the same terms that the other phenomena involving null subject are.
\end{abstract}

\section{The Italian pronominal sys- tem}

In Italian, there are two pronominal systems, characterized by a different syntactic distribution: weak pronouns, that must always be cliticized to the verb (e.g. la, lo, li, le - respectively her, accusative; him, accusative; them, masculine, accusative; them, feminine, accusative or her, dative), and strong pronouns (lui, lei, loro respectively he or him; she or her; they or them). The null subject can be considered as belonging to the system of weak pronouns. Notice that in Italian there is no neuter gender: nouns referring to inanimate objects are masculine or feminine. The weak pronouns used in this case are those of the corresponding gender, while, when a strong pronoun has to be used, paraphrase or deictics

*'This research was supported by DARPA grant no. N0014-85-K0018. are preferred. A strong pronoun for inanimate objects does exist - esso for masculine, essa for feminine, but it is not much used in current Italian.

Weak and strong pronouns are often in complementary distribution, as the following example shows - the contrast is between the use of the null or overt pronoun in subject position ${ }^{\mathbf{1}}$ :

Ex. 1 a) Quando Carlo $i$ ha incontrato Mario $_{j}$, When Carlo $\mathrm{C}_{i}$ has met Mario ${ }_{j}$ $\phi_{i / * j}$ non $\mathrm{gli}_{* i / j}$ ha nemmeno detto "ciao". he $_{i / * j}$ not to-him ${ }_{i / * j}$ has even said "hi".

b) Quando Carlo ha incontrato $\mathrm{Mario}_{j}$, When Carlo, has met Mario ${ }_{j}$, lui ${ }_{* i / j}$ non $\mathrm{gli}_{i / * j}$ ha nemmeno detto "ciao". he $_{* i / j}$ not to-himi h $_{* j}$ has even said "hi".

Notice the difference between sentences a and $b$ : in a the null pronoun in subject position refers to Carlo and therefore gli has to refer to Mario; in $b$ reference is switched. The overt pronoun lui in subject position requires its referent to be Mario, and therefore gli has to refer to Carlo.

There are some syntactic accounts of coreference phenomena in Italian, for example Calabrese's [Cal86]. He starts from the observation that weak pronouns are used in all those contexts in which there is an expected referent for the pronoun itself; he claims that we cannot use strong pronouns in place of weak ones, and vice versa ${ }^{2}$.

\footnotetext{
${ }^{1} \phi$ indicates a null subject and can be translated as an unstressed pronoun in English. In all the examples I will be using, if a proper name ends in $-o$ or $-i$, it has a male referent; if it ends in $-a$, a female referent. The translations I provide are literal and generally word by word.

${ }^{2}$ Actually Calabrese classifies pronouns as unstressed / stressed, and not as weak / strong, but I think his terminology may lead the reader to a wrong conclusion. In fact, while the "unstressed" pronouns can never be stressed, the "stressed" pronouns can, but not necessarily are.
} 
To formalize the concept of expected referent, he resorts to the notion of Thema, defined as the subject of a primary predication, where $x$ is a primary predicate of $y$ iff $x$ and $y$ form a constituent which is either $\theta-$ marked or [ + INFL]. He then says that a pronoun in position of Thema is expected to have another Thema as antecedent, and that if this coindexing occurs, the pronoun must be a weak one.

Through these definitions and rules he manages to account for a wide range of data, as far as single sentences are concerned, but when he tries to extend them to discourse, their usefulness and predictive power is not sufficient, and sometimes they give the wrong prediction. This is partly due to his very simplistic view of discourse, which he considers as a conjunction of sentences. Even for those sentences in which this view is sufficient, the argument that coreference depends only on the syntactic structure of the discourse and that we cannot use a weak pronoun when the theory predicts that a strong one is expected does not hold. Consider the following example:

\section{Wx. 2 D1) a) Ieri Carlo i ha incontrato Mario ${ }_{j}$.} Yesterday Carlo $i$ has met Mario ${ }_{j}$.

b) $\phi_{i / * j}$ Non $\mathrm{gli}_{4 i / j}$ ha nemmeno detto "ciao" $\mathrm{He}_{i}$ not to-him ${ }_{j}$ has even said "hi".

D2) a) Ieri Carlo i ha incontrato Maria ${ }_{j}$. Yesterday Carlo; has met Maria . $_{\text {. }}$

b) $\phi_{* i / j}$ Non gli $_{i / * j}$ ha nemmeno detto "ciao". She $_{j}$ not to-him ${ }_{i}$ has even said "hi".

Calabrese's analysis correctly explains the allowed and disallowed coreferences in D1: Mario is not the Thema of D1.a. So, if we want to have the subject of D1.b refer to Mario, we cannot use a weak pronoun, but we have to use a strong one: in fact, if we do use a null subject, it is interpreted as referring to Carlo.

Let's now consider D2. The structure of the iwo discourses is exactly the same. Therefore the theory predicts that, if we want to refer to Maria, which is not the Thema of D2.a, we have to use a strong pronoun, and not a null one: instead, D2.b is almost perfect.

The reason is that in D2.b the null subject has two potential referents, one male and the other female. While processing the sentence, the possibility that the null subject refers to Carlo is ruled out when the clitic gli, marked for masculine, is found. In fact, gli has to refer to Carlo; given that gli is not reflexive, it cannot corefer with the subject, therefore the latter is forced to refer to Maria.

This kind of disambiguation cannot be performed in D1.b, in which the null subject has two potential referents of the same gender.

I should mention that Calabrese, at the beginning of his paper, says that such features lgender, number and person] allow a first selection among the possible referents which are assigned to the pronominal. Presumably he would use these features as a superimposed filter to be applied to the whole sentence after it has been completely read or heard.

However, this could hardly fit in a model of how people process discourse: it is very likely that the normal human mode of operation is incremental [Ste89]. My claim is that disambiguation clues have to be taken into account as soon as they are available while processing a sentence. We will see in fact that they can help to make a discourse coherent or not according to their position in the sentence.

Notice that the issue here is to account not so much for the grammaticality or ungrammaticality of a sentence, as pure syntactic accounts do, but for more or less coherence in a discourse: this is exactly the purpose of centering theory. In particular, centering relates discourse coherence with the inference load that a certain sequence of utterances, and especially a certain choice of referring expressions, requires on the part of the hearer.

In the next section, I'll show how centering theory can be useful to explain certain uses of Italian pronouns in discourse, and in turn, how a richer pronominal system can help to refine the rules that centering uses.

\section{Centering theory}

It is now widely accepted that discourse is divided into segments (see for example [Web88]); a discourse is coherent when its constituent segments exhibit both local coherence - namely, coherence among the utterances of each individual segment, and global coherence - namely, coherence among the different segments.

Centering is an account of local coherence: it tries to determine the entity which an utterance most centrally concerns. Besides, it assesses the 
coherence of a discourse in terms of the different moves that a speaker can do (basically, going on to talk about the same entity or switching to another one), and in terms of how these moves are encoded, in particular as far as the choice of referring expressions is concerned. According to [GJW86], discourse coherence is a measure of the inference load a certain discourse imposes on a hearer. Notice that the view I am taking on centering is as a theory of discourse production. From [GJW86], it is not very clear whether centering concerns the production or the comprehension of discourse.

More technically, there are three moves that a speaker can perform, for every triple of utterances $U_{n}, U_{n+1}, U_{n+2}$, belonging to the same segment:

\section{DEF. 1}

Continuation: $U_{n}$ and $U_{n+1}$ concern the same entity; it is likely that $U_{n+2}$ will concern it too.

Retention: $U_{n}$ and $U_{n+1}$ concern the same entity, but it is not likely that $U_{n+2}$ will concern it.

Shifting: $U_{n}$ and $U_{n+1}$ concern different entities.

To formalize these concepts, the theory defines as centers those entities that serve to link one utterance to another in the same segment; an utterance $U_{n}$ typically has a single backward looking center $X(\mathrm{Cb})$, and a set of forward looking centers (Cf's) $\left\{Y_{1}, \ldots, Y_{m}\right\}$.

$\mathrm{X}, Y_{1}, \ldots, Y_{m}$ are all candidates for being $\mathrm{Cb}\left(U_{n+1}\right)$ (in fact $\mathrm{X}=Y_{i}$, for some $i$ ), and $\mathrm{Cb}\left(U_{n+1}\right)$ will be constrained to belong to the set of Cf's of $U_{n}$. Both $\mathrm{Cb}\left(U_{n}\right)$ and the set of Cf's $\left(U_{n}\right)$ correspond to linguistically realized NPs in $U_{n}$.

The set of Cf's for a given utterance $U_{n}$ is partially ordered; the ordering relation is affected by syntactic factors. In [GJW86], the only syntactic element that is identified in this respect is the subject of $U_{n}$ : it is the most likely entity to be $\mathrm{Cb}\left(U_{n+1}\right)$, therefore it is the highest ranked $\mathrm{Cf}$ in $U_{n}$. This assumption is definitely plausible, but it does not say anything about ordering among the other Cf's. For a more detailed analysis of the factors affecting Cf's ordering, see Kameyama's application of centering to Japanese [Kam85], and for more recent work on this topic, [WIC90]. I will not address this problem in the current paper.

Given that the $\mathrm{Cb}$ corresponds to the entity that an utterance concerns, the speaker has some choices as far as encoding the $\mathrm{Cb}$ goes. In [GJW86] the following rule $\mathrm{R} 1$ is proposed:

in $U_{n+1}$ the speaker can use

- a single pronoun, and that is the $\mathrm{Cb}\left(U_{n+1}\right)$;

- zero or more than one pronoun: then $\mathrm{Cb}\left(U_{n+1}\right)$ is

$-\mathrm{Cb}\left(U_{n}\right)$ if $\mathrm{Cb}\left(U_{n}\right)$ is realized in $U_{n+1}$,

- otherwise the highest ranked $\operatorname{Cf}\left(U_{n}\right)$ which is realized in $U_{n+1}$.

In order to ensure a coherent discourse, the speaker has to apply the following rule R2 as well:

Given $\mathrm{Cb}\left(U_{n}\right)=X, \mathrm{Cf}\left(U_{n}\right)=\left\{Y_{1}>\ldots>Y_{m}\right\}$, $\mathrm{X}=Y_{k}$, for some $\mathrm{k}, 1 \leq \mathrm{k} \leq \mathrm{m}$ :

if there are pairs $\left\{Y_{i}, Y_{j}\right\}$, with $i<j$, s.t. both $Y_{i}$ and $Y_{j}$ are realized in $U_{n+1}$, and if $Y_{j}$ is realized with a pronoun, then $Y_{i}$ has to be realized with a pronoun.

The previous rule requires that a speaker, if $s /$ he chooses to use a pronoun to refer to a certain $\mathrm{Cf} Y_{j}$, has to use a pronoun to refer to all the other Cfs realized in the current utterance and higher in the ordering than $Y_{j}$.

This rule accounts for the unacceptability of discourses like (from [GJW86]) ${ }^{3}$ :

Ex. $\left.3 U_{1}\right) \mathfrak{J} \mathrm{ohn}_{i}$ wanted to go for a ride yesterday. $\operatorname{cf}\left(U_{1}\right)=\{\mathrm{Johm}\}$

$\left.U_{2}\right) \mathrm{He}_{\text {i }}$ called up Mike . $_{\text {. }}$ $\mathrm{Cb}\left(U_{a}\right)=\mathrm{John}$ $\operatorname{Cf}\left(U_{z}\right)=\{$ John $>$ Mike

$\left.U_{3}\right) \mathbf{H e}_{j}$ was annoyed by John ${ }_{i}$ 's call.

In $U_{3}$, Mike is referred to with a pronoun; Mike was less highly ranked than John as a Cf, therefore, if we want to refer to John in $U_{3}$, we should also use a pronoun. The fact that in $U_{3}$ the proper name John is used makes the sequence unacceptable: in fact substituting his to John's results in an acceptable discourse.

\footnotetext{
${ }^{3}$ Notice that the first utterance of a discourse does not have a $\mathrm{Cb}$.
} 
After recognizing what $\mathrm{Cb}\left(U_{n+1}\right)$ is, the hearer can derive the kind of move that the speaker has performed in the following way:

\section{DEF. $1^{\prime}$}

Continuation: $\mathrm{Cb}\left(U_{n+1}\right)=C b\left(U_{n}\right)$ and $\mathrm{Cb}\left(U_{n+1}\right)$ is the most highly ranked element in $\operatorname{Cf}\left(U_{n+1}\right)$.

Retention: $\mathrm{Cb}\left(U_{n+1}\right)=C b\left(U_{n}\right)$ but $\mathrm{Cb}\left(U_{n+1}\right)$ is not the most highly ranked element in $\mathrm{Cf}\left(U_{n+1}\right)$.

Shifting: $\operatorname{Cb}\left(U_{n+1}\right) \neq C b\left(U_{n}\right)^{4}$.

Notice the correspondence between Def. 1 and Def. 1': the notion of $U_{n}$ and $U_{n+1}$ concerning the same entity corresponds to $U_{n}$ and $U_{n+1}$ having the same $\mathrm{Cb}$. The notion of $U_{n+2}$ going on to concern still the same entity corresponds to $\mathrm{Cb}\left(U_{n+1}\right)$ being the most highly ranked $\operatorname{Cf}\left(U_{n+1}\right)$.

\section{Centering and Italian pro- nouns}

I now want to recast the choices that the two Italian pronominal systems offer to a speaker in terms of centering, and, at the same time, refine centering itself. I will get evidence from examples like the following ${ }^{5}$ :

Ex. $\left.4 U_{1}\right)$ Maria $i$ voleva andare al mare.

Maria $_{i}$ wanted to go to the seaside.

$\left.U_{2}\right) \phi_{i}$ Telefono' a Giovanni ${ }_{j}$.

She $_{i}$ called Giovanni ${ }_{j}$ up.

$\left.U_{3}\right)$ a) $\phi_{i} \mathrm{Si}$ arrabbio' perche' $\phi_{i}$ non $\mathrm{lo}_{j}$ trovo' a casa.

She $_{i}$ got angry because she $e_{i}$ not him him $_{j}$ find at home.

b) $\phi_{i / ? j}$ Si arrabbio' perche' $\phi_{j}$ stava dormendo.

She $_{i} /$ ?He $_{j}$ got angry

because he ${ }_{j}$ was sleeping.

c) Lui ${ }_{j}$ si arrabbio' perche' $\phi_{j}$ stava dormendo. $\mathrm{He}_{j}$ got angry because he $\mathrm{e}_{j}$ was sleeping.

d) $\phi_{j}$ Si e' arrabbiatO perche' $\phi_{j}$ stava dormendo.

\footnotetext{
${ }^{4}$ Other versions of centering provide for two different types of shifting [BFP87].

${ }^{5} \mathrm{I}$ am using referents of different gender, because I want to show how gender and morphological markings come into play when resolving reference. Notice that these examples would not be ambiguous in English, given that null subject is not an option available to a speaker: the subject he/she would unambiguously pick up its referent.
}

$\mathrm{He}_{j}$ has gotten angry(-masc.)

because he $e_{j}$ was sleeping.

Various interesting facts come out from the four $U_{3}$ variations ${ }^{6}$ :

[a] The null subject refers to Maria, who, according to the rules in the previous section, is $\mathrm{Cb}\left(U_{3} . a\right)$, and the highest ranked element in $\operatorname{Cf}\left(U_{3} \cdot a\right) . U_{3} \cdot a$ thus demonstrates center continuation. The discourse is perfectly coherent.

[b] The most natural interpretation is that the null subject in the main clause refers to Maria - the null subject in the subordinate clause is forced to refer to Giovanni on pragmatic grounds.

However, for this same pragmatic reason, on second thought the null subject in the main clause may be interpreted as referring to Giovanni, but the discourse sounds less coherent.

[c] The speaker performs a felicitous center shifting by referring to Giovanni with an overt pronoun, given that Giovanni was not $\mathrm{Cb}\left(U_{2}\right)$, and not even the highest $\mathrm{Cf}\left(U_{2}\right)$.

[d] Contrast this utterance with [b]. They should have the same effect on the hearer, namely, the null subject should be interpreted as referring to Maria: instead in [d] it is felicitously interpreted as referring to Giovanni. This happens because in [d] the verb is in the present perfect tense ${ }^{7}$; the past participle agrees with the subject, and its masculine morphology forces the referent of the null subject to be Giovanni, and not Maria.

It seems to me that Ex.4 and other similar examples point to the following generalizations:

- typically, the speaker encodes center continuation with a null subject. This agrees with Kameyama's analysis of Japanese [Kam85]);

\footnotetext{
${ }^{6}$ As a warning to the reader, notice that $I$ am not worrying about the interpretation of the null subject in the subordinate causal clause, as it does not affect the interpretation of the null subject in the main clause, and it is affected by pragmatic reasons.

${ }^{7}$ The temporal relation between the preceding discourse and [d] is not right; $U_{2}$ should also be in the past perfect. However, this temporal incoherence does not affect resolution of pronoun reference.
} 
- he typically encodes center retention or shift with a stressed pronoun;

- he can felicitously use a null subject in cases of center retention or shift if he provides $U_{n+1}$ with syntactic features that force the null subject to refer to a particular referent and not to $\mathrm{Cb}\left(U_{n}\right)$.

My claim is that it is the syntactic context up to and including the verbal form(s) carrying tense and / or agreement that makes the reference felicitous or not. Consider $U_{3} . \mathrm{d}$ again: it is the fact that the main verb is marked for masculine that allows the null subject to refer to something different from $\mathrm{Cb}\left(U_{2}\right)$.

Analogous considerations hold for D2.b in Ex. 2. There the clitic gli precedes the verb and forces the null subject to refer to Maria. The fact that the clitic precedes the verb is crucial: evidence for this derives from examples involving modal verbs and clitics.

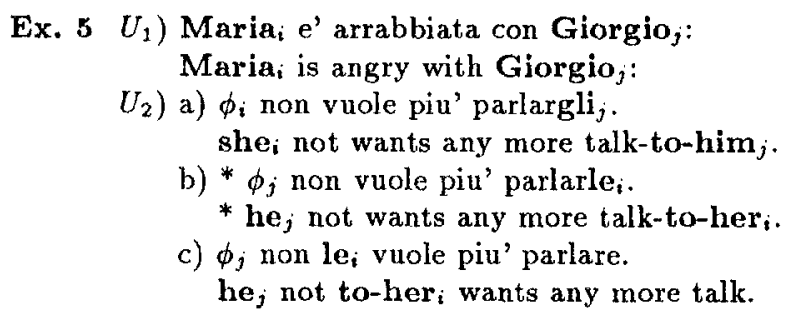

Here $U_{2}$.a is perfect, with the null subject referring to the higher $\operatorname{Cf}\left(U_{1}\right)$, namely Maria.

$U_{2} . \mathrm{b}$ is incoherent: the null subject is interpreted as referring to Maria, but when the clitic le is found, at the end of the sentence, the hearer is forced to change interpretation. The effect is similar to a syntactic "garden path".

$U_{2} . c$ is acceptable, for the very reason that the clitic le, that in $U_{2} . b$ is cliticized onto parlare, climbs in front of the modal verb vuole: so the hearer is forced to exclude Maria as referent of the null subject. This happens early enough so that no "garden path" effect is registered.

\section{Other phenomena}

The predictions presented in the previous section are quite reliable, but there are some cases that are not taken into account.

\subsection{Purpose of an utterance}

Consider the following example:
Ex. $\left.6 U_{1}\right)_{\text {Luisa }}$ ha lasciato suo marito $_{j}$ : Luisa $_{i}$ has left her husband ${ }_{j}$.

$\left.U_{3}\right) \phi_{* i / j}$ picchiava i bambini e si ubriacava. he $_{j}$ used to beat the children and get drunk.

In this case, $C b\left(U_{2}\right)$ is Luisa's husband. $U_{2}$ is felicitous, although the speaker uses a null subject to achieve a shift and no syntactic clue forces the null subject not to refer to Luisa. It looks like it is the function of $U_{2}$, namely, explaining why Luisa left her husband, that licenses the use of a null subject in this case.

It may even be argued that this case is outside the purview of centering, which explicitly states that the referential phenomena accounted for are within a single segment: $U_{2}$ may belong to a new segment, possibly much longer than what is shown here, that explains why Luisa left her husband.

On the other hand, it seems to me that the concept of local coherence is not totally dependent on having two utterances belonging to the same segment. The transition to another segment may override centering predictions; nevertheless, the referential expressions found in the first utterance of the new segment may need to be accounted for in terms of the Cf's of the last utterance of the previous segment. This may be what happens in Ex.6, if indeed $U_{2}$ belongs to a new segment.

\subsection{Null subject referring to a whole discourse segment}

Reference to a whole discourse segment is generally achieved in Italian by means of questo / cio', both equivalent to this, but sometimes a null subject is used (on this topic, see [DiE89]):

Ex. 7 Questi grandi atleti sono illuminati dai These great athletes come under mass media ogni due, ogni quattro anni, the media light every two, every four years, e devono conquistare una medaglia and they have to win a medal lottando contro il mondo intero fighting against the whole world per guadagnarsi l'affet to della gente. to gain people's affection.

Mentre in altri sport (nel calcio soprattutto) In other sports (in soccer above all), l'amore, la celebrita', i denari love, fame, money sono quasi automatici, quasi obbligatori. are almost automatic, almost compulsory. 
$\phi \mathrm{E}^{\prime}$ giusto?

Is this fair?

In the preceding example, the null subject in the last utterance refers to the whole previous discourse: the fact that a null subject, namely, the pronoun with the least informative content, that should supposedly refer to an expected referent, can be used in such a way, is a phenomenon that deserves explanation.

In general, centering does not say anything about reference to discourse segments, and in fact it may again be argued that clausal reference has nothing to do with local coherence. This actually depends on the perspective from which we look at clausal reference: it is possible that entities corresponding to discourse segments are implicitly included in the Cf's set; or that they are available for reference, but they have a status different from the normal Cf's; or that they have a different status altogether, for example that they do not exist as centered entities until they are referred to for the first time [Web88].

In any of these three cases, a theory of discourse coherence should at least partly address the problem.

\section{Conclusions}

In this paper, I have shown how the context up to and including the verb helps in disambiguating the reference for a null subject.

Some topics for future research have been discussed in the previous section. Integrating the analysis of these phenomena with centering will shed some light on the whole phenomenon of reference.

Centering gives us a vintage point of view in looking at local coherence in discourse as embodied by the choice of referring expressions that a speaker uses. Languages with richer morphological marking and agreement system than English can be very useful both to assess centering and to refine its rules.

\section{Acknowledgements.}

I would like to thank Prof. Bonnie Webber for her support and her comments on earlier versions of this paper, and Prof. Aravind Joshi for useful discussions.

\section{References}

[BFP87] Susan Brennan, Marilyn Walker Friedman, and Carl Pollard. A centering approach to pronouns. In Proc. 25th Meeting, Association for Computational Linguistics, pages 155-162, 1987.

[Cal86] Andrea Calabrese. PRONOMINA - Some properties of the Italian pronominal system. In N. Fukui, T. Rapoport, and E. Sagey, editors, $M I T$ Working papers in Linguistics. $P a$ pers in Theoretical Linguistics. Vol. 8 , 1986.

[DiE89] Barbara Di Eugenio. Clausal reference in Italian. In Proceedings Penn Linguistics Colloquium, 1989.

[GJW86] B. Grosz, A. Joshi, and S. Weinstein. Towards a computational theory of discourse interpretation. 1986. Unpublished manuscript.

[Kam85] Megumi Kameyama. Zero anaphora: the case of Japanese. PhD thesis, Stanford University, 1985.

[Ste89] M. Steedman. Grammar, interpretation and processing from the lexicon. In W. Marslen-Wilson, editor, Lexical Representation and Process, MrT Press, 1989.

[Web88] Bonnie Webber. Discourse deixis and discourse processing. Technical Report MS-CIS-88-75, Department of Computer and Information Science, University of Pennsylvania, 1988.

[WIC90] Marilyn Walker, Masayo Iida, and Sharon Cote. Centering in Japanese discourse. In Proc. COLING 90, 1990. 